\title{
Models of Collapsing Clouds and Star-Forming Regions as Analogs of the Solar Nebula
}

\author{
Paola Caselli \\ INAF-Osservatorio Astrofisico di Arcetri, Largo E. Fermi 5, I-50125 \\ Firenze, Italy
}

\begin{abstract}
To fully understand the formation and the evolution of the Solar Nebula, the physical and chemical characteristics of the "pre-solar" cloud should be known. Here, I review recent findings on the study of starless and pre-stellar cores, pointing out the importance of molecular freeze out in the chemical and dynamical evolution of star forming regions.
\end{abstract}

\section{Introduction}

Stars like our Sun form inside gas and dust condensations of molecular clouds, called dense cores. These objects are observed throughout our Galaxy, but particular attention is devoted to nearby $(<200 \mathrm{pc})$ dense cores, which are embedded in molecular cloud complexes, such as Taurus and Ophiuchus (e.g. Myers 1999; Evans 1999). Their proximity to Earth makes them the ideal targets on which to carry out accurate and detailed studies.

It is well known that roughly one half of the nearby dense cores are associated with young stellar objects (or protostars; Beichman et al. 1986), which actively interact with the surrounding medium, altering the chemical composition and physical structure of the parent cloud with their strong collimated winds (bipolar outflows) and radiation. The other half of dense cores are starless and, although some of them may be in equilibrium and never form a star (see, e.g., Lada et al. 2003), a fraction (recently estimated to be $\sim 5 \%$; Crapsi et al., in prep.) is thought to be on the verge of star formation, with clear evidence for centrally concentrated density profiles (e.g. Ward-Thompson et al. 1999) and contraction (Tafalla et al. 1998; Williams et al. 1999; Caselli et al. 2002a). Any information on the physical and chemical structure of these objects, called pre-stellar cores, will provide clues on the initial conditions of the star formation process and the material composition out of which stars as well as circumstellar disks, planets and comets will form.

Here, I will review recent progress in the study of starless and pre-stellar cores, the initial conditions in the process of star formation. Their chemical and physical properties will be described in an attempt to link "pre-solar" clouds to star-disk systems, for a better understanding of Solar Nebula evolution.

\section{From starless to protostellar cores}

In their evolution toward the formation of a young stellar object, dense cores with sizes of about $0.1 \mathrm{pc}$ and molecular hydrogen number density of a few $\times$ 
$10^{4} \mathrm{~cm}^{-3}$ (e.g. Caselli et al. 2002b), change their density distribution until the so-called "pivotal" state is reached (e.g. Shu et al. 1987). This stage is considered the starting point for protostellar accretion and evolution.

There is much debate nowadays about how the "pivotal" stage is reached. The classical picture (see e.g. Ciolek \& Mouschovias 1995) is that of a system contracting quasi-statically through ambipolar diffusion (the contraction motion of neutral particles in dense cores of low ionization, relative to the magnetic field and the ionized component). Another school of thought is represented by Hartmann et al. (2001; see also Mac Low \& Klessen 2004), who claim that cloud cores form faster than predicted by ambipolar diffusion models and that star (and cloud) formation takes place in a few (and not tens of) million years, due to the presence of supersonic turbulence generated by big explosions (such as supernovae). The two pictures imply different chemical and physical histories, so that it is crucial to understand which model is closer to reality. This is currently under investigation.

A series of detailed studies of starless cores, based on observations of molecular lines, have been carried out in the past few years (e.g. Willacy et al. 1998; Caselli et al. 1999; Bergin et al. 2002; Caselli et al. 2002a,c; Tafalla et al. 2002; Lee et al. 2003). From these studies, coupled with millimeter continuum dust emission observations (e.g. André et al. 2000), it is now clear that the sample of starless cores is not homogeneous and an evolutionary trend is present.

Some starless cores (e.g. B68, L1512) appear quiescent and close to hydrostatic equilibrium (Lada et al. 2003; Lee et al. 2003), with central densities of a few times $10^{5} \mathrm{~cm}^{-3}$, and no evidence of contraction. They appear to be "chemically evolved", with large abundances of "late-time" molecules (such as $\mathrm{NH}_{3}$ and $\mathrm{N}_{2} \mathrm{H}^{+}$) and clear signatures of molecular (mainly $\mathrm{CO}$ and $\mathrm{CS}$ ) freeze out. Other objects (e.g. L1521E) have similar density structures but are chemically young (Hirota et al. 2002), suggesting that they are recently formed.

Another category of starless cores is represented by objects such as L1498 and L1517B, which present a more complex kinematic structure, likely due to material accretion onto the core and core contraction (Tafalla et al. 2002). They have central densities of $\sim 10^{5} \mathrm{~cm}^{-3}$ and constant temperatures of $\sim 10$ $\mathrm{K}$. CO and CS disappear from the gas phase at gas densities of a few $\times 10^{4}$ $\mathrm{cm}^{-3}, \mathrm{~N}_{2} \mathrm{H}^{+}$maintains a constant abundance throughout the core, whereas the $\mathrm{NH}_{3}$ abundance increases toward the center (Tafalla et al. 2004). The different amount of depletion for $\mathrm{CO}$ and the two $\mathrm{N}$-bearing species can be reproduced by gas-grain chemical models which account for the different binding energies of $\mathrm{CO}$ and $\mathrm{N}_{2}$ (the common parent species of $\mathrm{N}_{2} \mathrm{H}^{+}$and $\mathrm{NH}_{3}$ ) onto dust grain surfaces (Bergin \& Langer 1997). The differing behavior of $\mathrm{N}_{2} \mathrm{H}^{+}$and $\mathrm{NH}_{3}$ is still not fully understood, although very low binding energies of ammonia precursors help in reproducing the observed trend (Caselli \& Aikawa, in preparation).

Other objects (e.g. L1544, L1521F) seem to be close to the "pivotal" stage, with about ten times larger central densities, more centrally concentrated density profiles, and with clear signs of contraction in the central few thousand AU (Caselli et al. 2002c; Crapsi et al., in prep.). These condensations, being on the verge of star formation, should be more properly called pre-stellar cores. They stand out in having large degrees of $\mathrm{CO}$ depletions and deuterium fractionations (see also Bacmann et al. 2003), and show line broadening in the central few 
thousands AU, likely due to inward motions (Caselli et al. 2002c). The recent strong detection of $\mathrm{H}_{2} \mathrm{D}^{+}$in the central $\sim 2500 \mathrm{AU}$ of L1544 (Caselli et al. 2003) had strong implications for the chemical structure of pre-stellar cores. In fact, the large $\mathrm{H}_{2} \mathrm{D}^{+}$abundance $\left(\left[\mathrm{H}_{2} \mathrm{D}^{+}\right] /\left[\mathrm{H}_{2}\right] \simeq 10^{-9}\right)$ inferred from observations is consistent with the central few thousands $\mathrm{AU}$ being completely deprived of species heavier than helium.

Therefore, the material out of which the "protostellar nebula" will form is essentially composed of $\mathrm{H}_{2}$ and dust grains covered with thick icy mantles. Assuming "classical" dust particles with radius $0.01 \mu \mathrm{m}$ and $10^{6}$ sites for adsorption of gaseous species (e.g. Tielens \& Allamandola 1987), it is easy to show that a complete freeze out of molecules implies dust grains covered by about one hundred layers of ice. The consequent increase in grain size has consequences for the dust properties, including the emissivity (Ossenkopf \& Henning 1994). In particular, thick icy layers are expected to boost grain coagulation (Ossenkopf 1993), so that large and fluffy aggregates probably form before becoming part of the protoplanetary disk. How this affects the evolution of the Solar Nebula is unknown and it awaits further investigation.

Interestingly, very young protostars (the so-called Class 0 sources; André et al. 1993) appear to have chemical characteristics (and kinematics) similar to pre-stellar cores, including molecular depletion (Belloche et al. 2002), reinforcing the idea that pre-stellar cores are indeed evolving toward stellar birth and can be considered as initial conditions in the process of star formation. Moreover, Class 0 sources possess a disk (see also Harvey et al. 2003), embedded (and formed) in the molecular depleted region. Finally, the presence of a rich chemistry on dust grain surfaces during the pre-stellar phase of evolution of a molecular cloud core is testified to by the recent discovery of complex organic molecules (hard to form in the gas phase) in the warm $(\sim 100 \mathrm{~K})$ gas surrounding the Class 0 source IRAS 16293-2422 (Cazaux et al. 2003). Molecular species such as ethyl cyanide $\mathrm{C}_{2} \mathrm{H}_{5} \mathrm{CN}$ are thought to be formed on grain surfaces during the cold pre-stellar phase and, after protostellar formation, released in the gas phase. These findings furnish stringent constraints on current chemical models of protoplanetary disks.

\section{References}

Andre, P., Ward-Thompson, D. \& Barsony, M. 1993, ApJ, 406, 122

Andre, P., Ward-Thompson, D. \& Barsony, M. 2000, in Protostars and Planets IV, ed. V. Mannings, A. P. Boss, \& S. S. Russel (Tucson: Univ. Arizona Press), p. 59

Bacmann, A., Lefloch, B., Ceccarelli, C., Steinacker, J., Castets, A. \& Loinard, L. 2003, ApJ, 585, L55

Beichman, C. A., Myers, P. C., Emerson, J. P., Harris, S., Mathieu, R., Benson, P. J. \& Jennings, R. E. 1986, ApJ, 307, 337

Belloche, A., Andre', P., Despois, D. \& Blinder, S. 2002, A\&A, 393, 927

Bergin, E. A., Alves, J., Huard, T. \& Lada, C. J. 2002, ApJ, 570, L101

Bergin, E. A. \& Langer, W. D. 1997, ApJ, 486, 316

Caselli, P., van der Tak, F. F. S., Ceccarelli, C. \& Bacmann, A. 2003, A\&A, 403, L37

Caselli, P., Walmsley, C. M., Tafalla, M., Dore, L. \& Myers, P. C. 1999, ApJ, 523, L165

Caselli, P., Walmsley, C. M., Zucconi, A., Tafalla, M., Dore, L. \& Myers, P. C. 2002a, ApJ, 565, 331 
Caselli, P., Benson, P. J., Myers, P. C. \& Tafalla, M. 2002b, ApJ, 572, 238

Caselli, P., Walmsley, C. M., Zucconi, A., Tafalla, M., Dore, L. \& Myers, P. C. 2002c, ApJ, 565, 344

Cazaux, S., Tielens, A. G. G. M., Ceccarelli, C., Castets, A., Wakelam, V., Caux, E., Parise, B. \& Teyssier, D. 2003, ApJ, 593, L51

Ciolek, G. E. \&, Mouschovias, T. Ch. 1995, ApJ, 454, 194.

Evans, N. J., II 1999, ARA\&A, 37, 311

Hartmann, L., Ballesteros-Paredes, J. \& Bergin, E. 2001, ApJ, 562, 852

Harvey, D. W. A., Wilner, D. J., Myers, P. C. \& Tafalla, M. 2003, ApJ, 596, 383

Hirota, T., Ito, T. \& Yamamoto, S. 2002, ApJ, 565, 359

Lada, C. J., Bergin, E. A., Alves, J. F. \& Huard, T. L. 2003, ApJ, 586, 286

Lee, J.-E., Evans, N. J., II, Shirley, Y. L. \& Tatematsu, K. 2003, ApJ, 583, 789

Mac Low, M. -M. \& Klessen, R. S. 2004, Rev. Mod. Phys., 76, 125

Myers, P. C. 1999 in The Physics and Chemistry of the Interstellar Medium, Proceedings of the 3rd Cologne-Zermatt Symposium, Eds.: V. Ossenkopf, J. Stutzki and G. Winnewisser \& G. Winnewisser, GCA-Verlag Herdecke, p. 227

Ossenkopf, V. 1993, A\&A, 280, 617O

Ossenkopf, V. \& Henning, Th. 1994, A\&A, 291, 9430

Shu, F. H., Adams, F. C. \& Lizano, S. 1987, ARA\&A, 25, 23

Tafalla, M., Mardones, D., Myers, P. C., Caselli, P., Bachiller, R. \& Benson, P. J. 1998, ApJ, 504, 900

Tafalla, M., Myers, P. C., Caselli, P. C., Walmsley, C. M. \& Comito, C. 2002, ApJ, 569, 815

Tafalla, M., Myers, P. C., Caselli, P. C. \& Walmsley, C. M. 2004, A\&A, 416, 191

Tielens, A. G. G. M. \& Allamandola, L. J. 1987, in Physical Processes in Interstellar Clouds, ed. G. E. Morfill \& M. Schloer (Dordrecht: Reidel), p. 333

Ward-Thompson, D., Motte, F. \& Andre, P. 1999, MNRAS, 305, 143

Willacy, K., Langer, W. D. \& Velusamy, T. 1998, ApJ, 507, L171

Williams, J. P., Myers, P. C., Wilner, D. J. \& di Francesco, J. 1999, ApJ, 513, L61 